\title{
Protée
}

\section{Organisations interobjectives et intersubjectivité dans les trains}

\section{Michela Deni}

Volume 29, numéro 1, 2001

La société des objets. Problèmes d’interobjectivité

URI : https://id.erudit.org/iderudit/030618ar

DOI : https://doi.org/10.7202/030618ar

Aller au sommaire du numéro

Éditeur(s)

Département des arts et lettres - Université du Québec à Chicoutimi

ISSN

0300-3523 (imprimé)

1708-2307 (numérique)

Découvrir la revue

Citer cet article

Deni, M. (2001). Organisations interobjectives et intersubjectivité dans les trains. Protée, 29(1), 75-83. https://doi.org/10.7202/030618ar

\section{Résumé de l'article}

Cet article présente une analyse des objets dont se compose l'aménagement intérieur des trains de passagers en Italie. On observe que les relations interobjectives suscitées par la disposition des sièges, des poignées, des interrupteurs, des tablettes, des cendriers, etc., créent des espaces signifiants différenciés selon les modèles de wagons. Une typologie en est proposée. L'usage que les passagers font de ces espaces est lié à la manière dont ils en interprètent les effets de sens, compte tenu de la présence et de la valeur relationelle de chaque élément. Cet ensemble de relations induit des formes d'inclusion et d'exclusion entre groupes de passagers. 


\section{O RG AN ISATIO N S IN TERO BJECTIVES ET INTERSU BJECTIVITÉ DANS LES TRAIN S*}
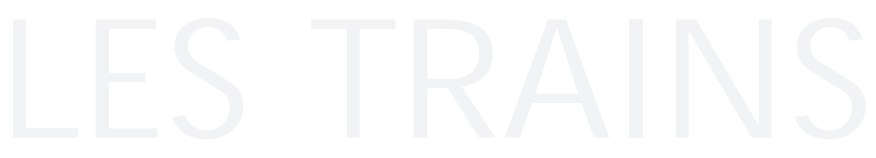

M ICHELA DEN I

Notre intervention part d'une réflexion sur l'organisation de l'espace à l'intérieur des trains de voyageurs et sur la disposition des objets qui composent l'équipement des wagons. Ces intérieurs nous permettent d'enquêter sur le rapport entre interobjectivité et intersubjectivité.

Dans l'analyse qui suit, la relation intersubjective est envisagée à partir de sa structuration sur la base de l'organisation interobjective. En d'autres termes, avant même de se concentrer sur la relation réciproque entre utilisateurs, ou entre objets et utilisateurs, l'analyse porte sur le niveau interobjectif. Comme nous le verrons, la dimension interobjective comporte une transformation des relations intersubjectives. Les objets, en effet, en raison même des relations interobjectives qu'ils entretiennent, construisent les espaces, et en même temps manipulent l'utilisateur et le dirigent vers des séquences d'action spécifiques et, par conséquent, modifient les interactions humaines. Toutes ces façons d'agir des objets mettent en évidence leur caractère factitif ${ }^{1}$. Parmi les objets qui promeuvent et garantissent des règles sociales, culturelles et morales moyennant une forme ou une autre de conditionnement proxémique, nous pouvons indiquer à titre d'exemples des dispositifs comme les compte-personnes dans les lieux publics, comme les fermeportes, faits pour ralentir le cours d'une porte qui se ferme toute seule et sans bruit $^{2}$, comme les accoudoirs de sièges dans les espaces publics, qui fonctionnent comme des marqueurs frontières ${ }^{3}$ garantissant le respect de l'espace personnel, et comme la forme des sièges ou des bancs qui prévoient des distances réciproques codifiées et des postures obligées (il suffit de penser aux bancs «anti-clochard» dont la forme empêche de se coucher). Sur le plan intersubjectif, ces objets modifient les comportements sans la nécessité de faire appel explicitement à des compétences sociales ou morales ${ }^{4}$. Leur action plus ou moins discrète - dont nous prenons conscience seulement lorsqu'ils ne fonctionnent pas - se traduit en des façons involontairement éduquées (on ne laisse pas les portes ouvertes et on ne les ferme pas bruyamment; on respecte l'espace des autres, etc.). Comme l'a montré Bruno Latour, il s'agit d'objets délégués qui, à travers une réification, soumettent l'action d'un actant humain à des restrictions imposées par l'objet.

* La présente étude reprend certains des résultats d'une étude plus détaillée incluse dans M. Deni,

Per una semiotica degli oggetti. La dimensione fattitiva, thèse de doctorat dirigée par U. Eco, Bologne, 1999. 


\section{OBJETS, ESPACES ET INTERACTIONS}

Tous les objets présents dans un lieu créent un contexte particulier du milieu. Comme dans tout processus de signification, il s'agit d'éléments qui n'ont pas de sens en eux-mêmes, mais qui transforment et augmentent leurs potentialités sémiosiques dans leur relation avec les autres objets. Ils prennent ainsi une identité axiologique à valeur relationnelle. C'est donc la relation syntagmatique des objets, résultat de leurs rapports réciproques (paritaires, hiérarchiques, positionnels) ${ }^{5}$, qui construit chaque espace et détermine l'interprétation de l'espace même. L'efficacité factitive, tout comme l'effet de sens global, dérive de la coprésence de différents objets qui perdent leur sens originaire et se recontextualisent en un nouveau système de signesobjets, prêt à construire des signifiés différents et plus complexes (ou simplifiés) par rapport aux systèmes d'origine.

Comme nous le verrons, un résultat concret de cet aspect peut se vérifier dans l'analyse de la disposition, de la forme et même de la relation entre les différentes parties des objets qui composent l'équipement des trains. De telles caractéristiques a) prescrivent des configurations proxémiques préconstituées, $b$ ) déterminent l'interprétation de l'espace, c) modifient le type de relations que les voyageurs entretiennent entre eux. La disposition des sièges, des tablettes, des cendriers et de tout autre objet agit sur le plan proxémique en présupposant la mise en acte d'une syntaxe générale de séquences d'action, qui influence les modalités personnelles du voyage et les possibilités d'interaction des voyageurs en transformant le train en espace topique (lieu d'action) ou en espace hétérotopique ${ }^{6}$ (lieu de suspension de toute action). Les objets statiques aussi bien que dynamiques, comme les fauteuils ou tous ces objets qui s'ouvrent, s'allongent, s'élargissent (comme les cendriers, les tablettes et les accoudoirs), ont sur ce plan des conséquences.

Comme tous les espaces publics et comme tous les non-lieux ${ }^{7}$, le train réunit dans le même espace des personnes inconnues, contraintes de voyager ensemble pour atteindre un endroit spécifique. À la différence de beaucoup d'autres espaces sociaux, le train prévoit une division intime de l'espace et impose certaines dispositions proxémiques, et ceci pendant des périodes qui peuvent être prolongées. Pour toutes ces raisons, l'organisation de l'espace dans le train doit permettre un afflux important de passagers et, en même temps, permettre à chacun d'eux de garder une certaine intimité. Normalement, l'espace individuel disponible est physiologiquement insuffisant, mais il est converti en espace culturellement adéquat par la compétence situationnelle des passagers. L'organisation des wagons est pensée de façon à garantir un milieu doté d'un certain charme ou d'un confort moyen: la disposition de l'équipement doit permettre aux passagers de lire, de se reposer, de parler entre eux ou de travailler. Naturellement, les caractéristiques de l'équipement changent selon le modèle du train, selon son parcours (bref, moyen ou long) et selon la classe (différences dans les tissus de revêtement, l'ampleur des sièges, la distance entre eux, les couleurs, etc.).

En observant la grande majorité des trains italiens à moyen et long parcours (Espresso, Interregionale (IR), wagon pullman de l'Intercity, ETR 450, ETR 500, ETR 480), on peut remarquer comment la disposition de l'espace tend à segmenter différentes zones signifiantes auxquelles sont attribuées des valeurs sémantiques particulières. Plusieurs trains sont formés par différents types de voitures et, quand cela est possible, les passagers tendent à s'installer dans certaines zones, les préférant à d'autres. Comme dans n'importe quel autre lieu, la disposition des objets (fauteuils, tablettes, etc.) prend un signifié précis et construit son propre utilisateur modèle ${ }^{8}$ en s'adaptant et en construisant ses propres exigences (repos, travail, socialité), le portant à un choix précis plutôt qu'à un autre. Ces considérations sont valables en particulier pour les passagers qui font la navette, qui connaissent l'organisation des voitures d'un train et qui élaborent des systèmes de préférences en fonction de leurs habitudes et de leurs exigences spécifiques. Mais c'est l'analyse même des dispositifs existants qui va nous permettre de vérifier que l'espace est bien une construction sémiotique qui produit du sens à travers les objets qui le forment ${ }^{9}$. 


\section{ORGANISATION DE L'ESPACE DANS LES TRAINS ITALIENS}

\section{L'Espresso}

La composition des trains Espresso prévoit en principe des voitures subdivisées en compartiments à six places, avec deux banquettes se faisant face, chacune à trois places indépendantes, à inclinaison réglable, séparées par des accoudoirs; les places aux extrémités des banquettes donnent évidemment sur la fenêtre ou sur le couloir (photos 1, 2, première et seconde classes). Une telle organisation implique que les passagers se trouvent les uns en face des autres, et très proches les uns des autres, même s'ils sont séparés par des accoudoirs. Le compartiment ressemble à un petit salon aménagé pour favoriser la conversation des passagers. La distance entre les passagers, intime ${ }^{10}$ sans aucun doute, oblige à une certaine attention, pour le moins en seconde classe, si l'on ne veut pas heurter les jambes de la personne assise en face ou toucher l'épaule ou le bras de celle assise à côté. La forme et la dimension du fauteuil, par exemple, présupposent une gamme limitée de positions, qui détermine de la part des passagers la mise en acte de pratiques implicites de négociation (contractuelle ou polémique) de l'espace: un passager qui tient sa jambe à droite implique que la personne en face tienne la sienne du côté opposé; si on garde les jambes fermées il est probable que la personne en face ouvre les siennes; si on appuie le bras sur l'accoudoir on empêche le voisin de le faire car c'est là un élément commun; un changement de posture chez un passager détermine un déplacement simultané chez ses voisins. C'est pour cela que le compartiment fermé est adapté aux voyages de groupes et aux contacts sociaux: la position des banquettes favorise en effet le contact, en particulier entre les personnes qui occupent la zone centrale et qui n'ont pas la possibilité d'un point de fuite. Au contraire, la place près de la fenêtre permet de déplacer le regard hors du compartiment et d'amplifier virtuellement son propre espace, tout comme la place près du couloir. La position centrale est à tous égards la moins intime et celle qui réduit le plus la latitude du regard puisqu'elle ne permet pas d'exclure les autres passagers du champ visuel, en sorte que les personnes qui sont au centre
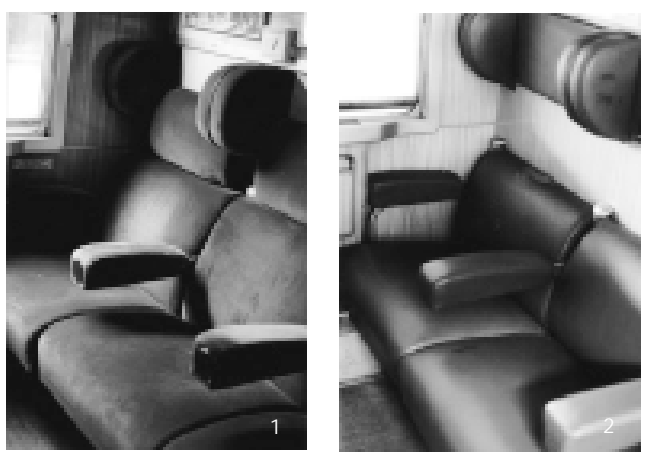
n'ont d'autre ressource, pour éviter de favoriser le contact, que de nier le regard d'autrui en lisant ou en fermant les yeux. Les trains à compartiments structurent toujours une organisation proxémique englobante, qui favorise les possibilités d'interaction focalisée en stimulant la conversation des passagers sociables; de la même façon, ils diminuent toute possibilité d'isolement pour qui y aspirerait ${ }^{11}$. L'espace intime et circonscrit exclut la possibilité de se dérober à l'interaction, sauf à se sentir proxémiquement inadapté puisque l'effet de sens du compartiment comme milieu «familial» et «social» est d'étiqueter, par opposition, comme impolie ou asociale, toute personne qui se soustrait volontairement à l'interaction collective - surtout si elle occupe la position centrale, car elle rompt le flux de la communication entre les autres passagers. Ainsi, sur le plan du contenu, nous pouvons définir le wagon à compartiments comme un espace privécollectif, puisque c'est un espace qui présuppose des pratiques d'inclusion entre le groupe des passagers présents et, en même temps, d'exclusion des passagers étrangers au compartiment.

Lorganisation de l'espace des compartiments fermés donne lieu à des proxèmes ${ }^{12}$ fréquents dans l'occupation des places, même lorsque le compartiment n'est pas complet: en général le premier espace occupé est celui près de la fenêtre, le second est celui près du couloir. Si deux passagers sont des personnes qui ne se connaissent pas, ils occuperont probablement l'espace près de la fenêtre et l'espace près du couloir de la banquette située en face. S'ils sont trois, c'est la place centrale d'une banquette et les deux places extrêmes de l'autre qui seront occupées pour s'assurer plus d'espace autour et devant, éviter les contacts physiques et la nécessité de négocier une installation plus commode. De telles stratégies sont fréquentes et répétées et sont réalisées à partir de l'organisation de l'espace: quand le premier passager s'assied au centre, il est probable qu'il n'a pas 
envie que quelqu'un d'autre occupe la même banquette. Il existe évidemment des stratégies que tout le monde utilise pour éviter que quelqu'un n'occupe la place à côté et qui consistent à disposer des objets personnels, les marqueurs centraux, sur les places contiguës, comme des sacs, des journaux et des vestes pour marquer le territoire de possession. Ces stratégies montrent bien une conscience implicite de la signification codifiée des comportements proxémiques et des valeurs sémantiques de l'espace.

Contrairement à ce que l'on pourrait penser, l'espace «fermé» du compartiment du train, du point de vue sémiotique, coïncide sur le plan du contenu avec un espace "ouvert», «social» et, plus précisément, "privé-collectif».

\section{Interregionale (IR)}

Les voitures des trains Interregionali sont ouvertes et non subdivisées en compartiments (photos 3, 4, 5). En seconde classe, les fauteuils sont disposés en deux files de chaque côté du couloir, alors qu'en première il y a une file de fauteuils uniques d'un côté, et de deux de l'autre. Chaque fauteuil en a un autre en face; cette disposition forme des microgroupes de sorte que l'espace est ouvert et collectif. Par rapport à d'autres trains, les fauteuils sont plus étroits et de structure rigide, le revêtement est en tissu ou en velours sur un rembourrage rigide et les accoudoirs sont de façon générale en plastique dur. De telles caractéristiques sont intéressantes puisque la position assise, bien que commode (dos droit et zone lombaire soutenue), n'invite pas au repos à cause de la rigidité. Les trains $I R$ font de fréquents arrêts et connaissent une affluence continue qui peut être une des causes du choix de ce type de sièges: la position assise y apparaît "temporaire", adaptée à de brèves périodes et non pas destinée au repos. L'équipement global est polychrome et les couleurs choisies (gris, rouge et noir), loin d'être chaudes et relaxantes, sont fortes et, dans l'ensemble, froides et discontinues. Autant la disposition des fauteuils que l'équipement produisent l'impression d'un lieu de passage semblable à une salle d'attente. De plus, l'espace, bien qu' "ouvert", suggère un rapport amical (bien que limité dans le temps) entre passagers, à la différence de ce qui se passe, comme nous le verrons, dans le wagon pullman des trains Intercity et dans les ETR. De plus, ici, l'espace global n'est pas subdivisé en zones individuelles, faute de barrières matérielles, de marqueurs frontières comme les tablettes: d'où la nécessité de pratiques de négociation proxémique. L'espace ouvert, le passage continu et l'organisation en microgroupes favorisent l'interaction entre les voyageurs qui fréquentent ce type de trains, facilitée certainement aussi par la répétition des mêmes parcours et des horaires communs.

Du point de vue sémiotique, l'organisation du milieu constituée par les objets de l'équipement crée l'effet d'un espace «anonyme» (formes anguleuses, rationnelles et couleurs froides, matériaux durs et lavables comme le plastique et le linoléum), "public» (espace ouvert) mais en même temps animé (couleurs contrastées, discontinues et peu harmonieuses comme l'orange, le gris, le noir et le rouge) et "collectif" (microgroupes centralisateurs). Comme on peut le remarquer, dans ce cas les oppositions individualisées sur le plan de l'expression "ouvert» versus «fermé», correspondantes à celles individualisées sur le plan du contenu «individuel» versus "collectif», ne sont pas suffisantes. Lorganisation spatiale, chromatique et positionnelle des éléments principaux de l'équipement ne produit pas le même système d'oppositions. Le train $I R$ configure, en effet, un espace vivant et informel qui peut être défini comme "semi-publiccollectif " grâce à une dimension qui n'est pas vraiment publique (on trouve des microgroupes inclusifs), mais accessible.

\section{Intercity (IC)}

La composition des trains Intercity prévoit des voitures à compartiments et des wagons ouverts. Comme toujours, la première classe a des compartiments plus spacieux, des fauteuils plus larges dotés d'une tablette que l'on peut extraire de l'accoudoir, de la moquette au sol, des fenêtres et des 

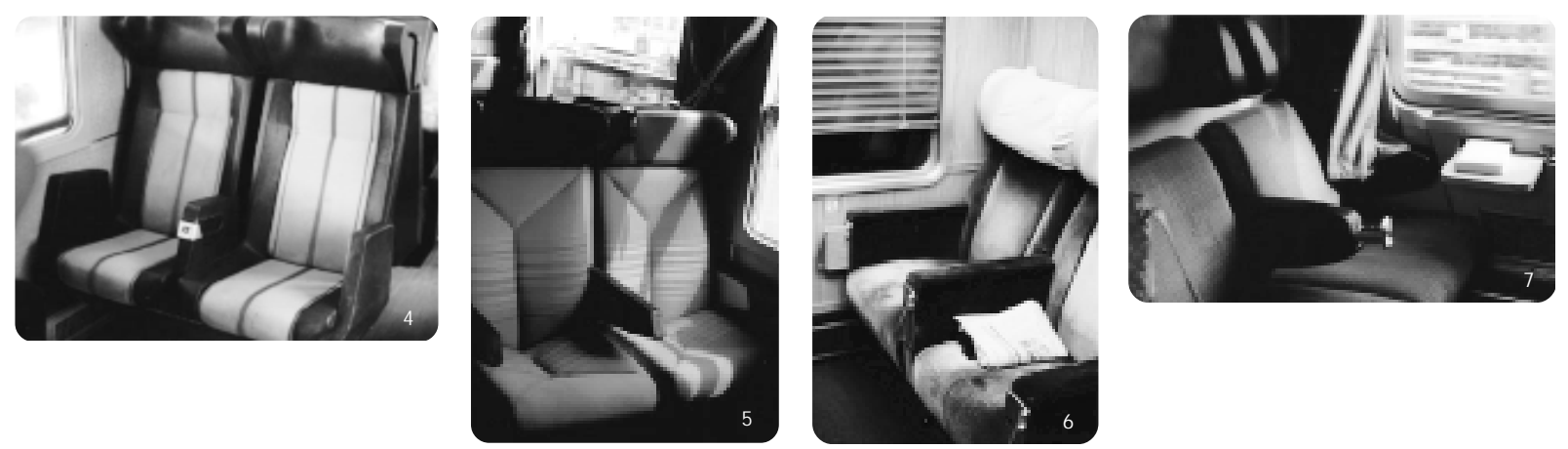

portes plus grandes, des stores automatiques (photo 6). Nous avons déjà considéré, en décrivant l'Espresso, la disposition spatiale et les conséquences proxémiques du compartiment fermé. Toutefois, dans ce cas-ci, l'effet de «salon» s'accentue, car ces compartiments sont plus soignés et plus accueillants (couleurs pastel, chaudes, claires, bon éclairage, matériaux légers et apparemment lavables), mais la sensation de bien-être augmente dans les voitures «Grand Confort» de première classe (meilleur éclairage, design plus soigné, couleurs neutres et reposantes comme le bleu ciel et le gris, stores électriques, portes coulissantes transparentes et grands miroirs). Encore une fois, l'espace en première classe est plus large et cela contribue à modifier la posture du voyageur qui, même dans un compartiment plein, peut adopter des positions commodes en évitant la continuelle négociation proxémique avec les voisins. L'effet de "milieu ouaté» dérive de la moquette et des tissus de revêtement qui «absorbent» les bruits et donnent une isolation acoustique apparemment meilleure que celle de la seconde classe. La connotation de "modernité», de «bien-être» et de "luxe», qui découle de ces particularités, induit une impression de relaxation qui tranche avec l'équipement "économique de base» et le "confort moyen» de la seconde classe (photo 7).

Les wagon pullman font partie de la composition des IC; ils possèdent des places spéciales, des boutons d'alarme et comportent des toilettes adaptées aux passagers handicapés. Ils ont été les premiers wagons à offrir des dispositifs technologiques apparemment innovateurs, même si cela correspond plus à un effet de sens qu'à la réalité. L'espace du wagon est divisé en deux par un panneau transparent, séparant le compartiment fumeurs (fauteuils rouges) de celui nonfumeurs (fauteuils bleus). Les sièges sont indépendants, à inclinaison réglable et disposés en quatre rangées séparées, deux à deux, par un couloir. Le dos de chaque fauteuil comporte une tablette que l'on peut extraire et un appuie-pieds. L'espace pour la position assise est moindre que celui des autres wagons, mais l'espace entre les fauteuils se faisant face est plus large. Les fauteuils vont deux à deux, séparés par un couloir, et dans la zone centrale il y a quatre microgroupes formés par quatre fauteuils avec une double tablette au centre, deux d'un côté et deux de l'autre. Les fenêtres, amples et continues, laissent entrer beaucoup de lumière. Du point de vue sémiotique, cette organisation spatiale produit et prescrit des configurations proxémiques complètement différentes de celles des wagons à compartiments. Comme nous l'avons déjà dit, le compartiment est un espace «fermé» qui, par son organisation en forme de salon, «ouvre» la dimension sociale. Le wagon pullman, au contraire, est un espace «ouvert» qui, paradoxalement, ne favorise pas le contact social. L'ampleur de l'espace, accentuée par le fait qu'il est délimité surtout par de vastes fenêtres, favorise encore plus l'effet d' "ouverture» et de «visibilité». Contrairement au compartiment fermé qui tend à faire se concentrer le regard à l'intérieur, ces voitures favorisent le décentrement du regard en donnant la possibilité de nombreux points de fuite. Le passager peut regarder en avant et voir toute la longueur du wagon; en regardant sur les côtés, il voit le panorama par l'ampleur des fenêtres. L'organisation spatiale ("ouverte») de ces voitures permet au voyageur un certain «renfermement» en lui-même, grâce à la possibilité de se soustraire à toute interaction obligée. Seules quelques places en effet imposent la relation face à face avec d'autres passagers (les quatre microgroupes) et, en ce cas, une tablette qui peut s'ouvrir les sépare. Les grandes fenêtres et la séparation nette de la tablette contribuent à l'intimité proxémique: chaque passager est séparé des autres par des accoudoirs et, devant lui, par la tablette qui, comme les accoudoirs, fonctionne en tant que marqueur frontière et délimite l'espace personnel. Ces 
éléments (le dossier derrière, la tablette devant, les accoudoirs sur le côté) entourent, protègent,
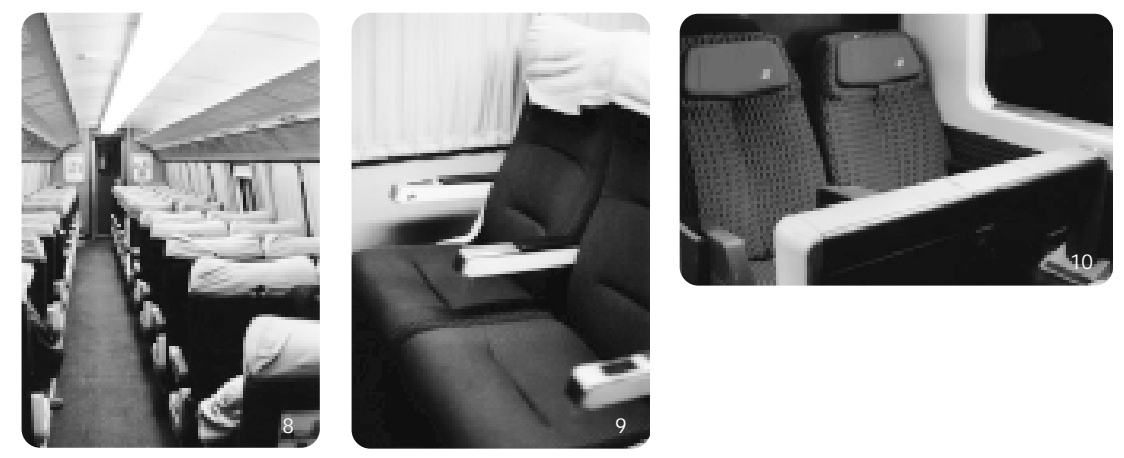
embrassent, mettent en évidence l'espace de chacun et imposent des configurations proxémiques précises, qui sont autant de moyens d'éviter la guerre proxémique ${ }^{13}$. De plus, les mêmes objets prennent en charge le parcours modal du simple voyageur par rapport aux autres passagers. Par exemple, sur le plan modal, les barrières matérielles formées par les objets entraînent un «devoir-être» et un «ne-paspouvoir-faire». Ainsi, l'espace «ouvert» du wagon pullman peut être défini comme un espace "publicindividuel ": malgré l'absence de compartiments, en effet, la disposition des objets exclut la possibilité d'accès aux autres passagers. Toutefois le wagon présente des modalités d'interaction différentes selon la place occupée. La banquette à quatre, munie d'une tablette, est adaptée à qui voyage en compagnie de quelqu'un, car elle favorise l'interaction, même si les barrières matérielles et l'espace ouvert détournent l'attention de l'espace-salon et peuvent décourager une interaction focalisée. Le wagon ouvert réunit en effet un grand nombre de personnes, mais décourage les approches interactionnelles entre inconnus et les échanges de conversation à voix haute, car il est possible de s'exposer à une sanction sociale de la part des autres passagers en raison de la visibilité totale de chaque place et de la dimension publique.

\section{ETR 450}

Ce train, créé par l'équipe de Giugiaro, est le plus vieux modèle des ETR. Les sièges, disposés en rangées de trois (deux d'un côté du couloir, une de l'autre), possèdent une tablette au dossier pour le passager qui occupe la place derrière (photos 8,9 ). Cependant, les voitures sont privées de la plupart des objets «technologiques» (écouteurs, boutons) présents dans les autres ETR, et l'organisation interne rappelle celle d'un avion: l'espace est entièrement utilisé pour la disposition des fauteuils, tous orientés vers l'avant, et les fenêtres semblent plus petites à cause de la présence de rideaux en tissu lourd toujours dépliés

grâce à un système à coulisse qui leur imprime une certaine rigidité. L'effet de sens global est celui d'un moyen de transport commode mais entièrement concentré sur le voyage, sans la moindre concession à une forme quelconque de distraction. La sobriété et la rigueur du ETR 450 montrent clairement que c'est là le lieu exemplaire d'un moyen fonctionnel de transport rapide, ce qui contribue à l'effet d'ennui chez les passagers, qui ont comme seul et unique but d'arriver le plus vite possible à destination. C'est un moyen impersonnel dont les couleurs et la «fermeture» spatiale délimitent un lieu rationnel et borné, n'offrant aucune zone qui puisse être explorée ou qui puisse éveiller la curiosité. Par exemple, les portes et les panneaux entre une voiture et l'autre sont fermés, il n'y a pas de boutons pour commander les différentes fonctions, ni d'objets particuliers à utiliser ou à observer; au contraire il y a des panneaux mystérieux -à «ne pas toucher»-, qui apparemment cachent des appareils techniques profondément différents des objets «friendly» qui invitent à l'utilisation et qui caractérisent les trains plus récents. Les autres ETR, en effet, grâce à la présence d'objets qui, dans la majeure partie des cas, se révèlent utiles (boutons, lumières, filodiffusione ${ }^{14}$, etc.), encouragent volontiers la possibilité de se délasser dans des espaces plaisants, éclairés de petites lumières et ressemblant à de grands salons. Cet ETR 450, en revanche, est un train claustrophobique, qui rappelle morphologiquement une galerie ou un gros tube avec ses fenêtres couvertes de rideaux, sa grande quantité de fauteuils, son mauvais éclairage et sa quasi totale monochromaticité.

L'organisation globale de l'espace interne de l'ETR 450 ne présente pas de configurations proxémiques favorisant la socialité, la curiosité pour le milieu extérieur ou intérieur et la relaxation. Dans ce cas, et bien que les voitures ne soient pas organisées en compartiments, la «fermeture» morphologique de l'espace (l'impression d'être dans un tunnel, parois 


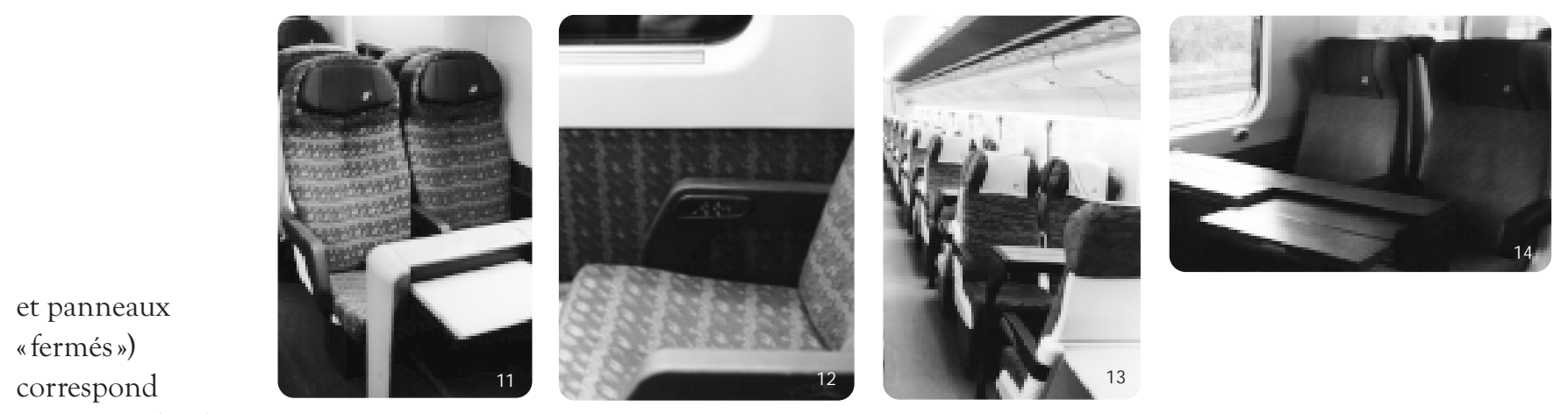

même, sur le plan

du contenu, à une «fermeture émotive» des passagers : c'est pour ces raisons que nous pouvons parler d'un espace «semi-privé-individuel» (un espace «privéindividuel» dans le train pourrait coïncider seulement avec les toilettes). La discontinuité évidente entre intérieur et extérieur fait de l'ETR 450 une forme exclusivement fonctionnelle au voyage («technologie», «modernité», «sobriété", « rationalité», "vitesse»), un espace hétérotopique où le passager se trouve momentanément, et qui contraste avec l'espace topique qui, dans ce cas, coïncide avec le lieu de départ et celui d'arrivée. C'est pourquoi le parcours effectué sur un ETR 450 représente un moment non social, sans plaisir, un temps suspendu et inutile mais nécessaire pour atteindre un lieu.

\section{ETR 500}

Il s'agit du train pendolino, réalisé en vert par Pininfarina. Les fauteuils sont généralement disposés comme des "points-salon", l'un en face de l'autre avec la double tablette au centre (photos 10, 11, 12); ou bien ils sont organisés en rangées doubles ou simples, et tout le monde regarde dans la même direction. Les accoudoirs des fauteuils sont aménagés pour offrir un écouteur et un choix de longueurs d'ondes sur la filodiffusione. Du point de vue sémiotique, l'organisation de l'espace de ce train n'offre pas la possibilité de configurations proxémiques très différentes de celles de l'ETR 480, auquel nous passons donc tout de suite.

\section{ETR 480}

L'ETR 480 de Giugiaro est le modèle le plus moderne des trains italiens. Il est composé de voitures ouvertes. À la seule observation des toilettes, on a l'impression d'un train fortement technologique (il possède de nombreuses touches automatiques), mais en même temps «humanisé». La disposition des fauteuils comprend et des points-salon centraux

(quatre fauteuils face à face, deux à deux, séparés par une double tablette) et quelques rangées de fauteuils tous orientés dans la même direction (photos 13, 14). Bien que l'ETR 480, comme les trains Interregionali, se caractérise par des voitures ouvertes, à la différence de ceux-là, chacun des passagers est ici doté d'une zone propre (inclusive us exclusive), comme c'est aussi le cas dans le wagon pullman de l'IC: les accoudoirs et les tablettes entourent et circonscrivent l'espace du voyageur qui peut toujours utiliser sa tablette personnelle. Dans ce cas aussi, la disposition proxémique des passagers, telle qu'elle est organisée par la disposition des fauteuils et des tablettes, renvoie à la fois à l'«ouvert» sur le plan de l'expression, et au «public-individuel» sur le plan du contenu. D'autres éléments accentuent cet effet de sens: les couleurs «reposantes» des tissus (bleu, gris léger) et des parois (très claires), les formes arrondies des fauteuils et des porte-bagages, l'absence d'angles, l'utilisation de plastique intégré dans un matériel plus mou et «chaud» comme la gomme. Ces détails contribuent à produire l'effet global de "modernité», de «technologie» et de "design anthropomorphe» et favorisent le relaxation. Chaque passager est incité à s'occuper de ses propres affaires (la tablette personnelle) même si le train est un moyen de transport public, et qui plus est, très fréquenté. L'isolement individuel est favorisé par la possibilité d'écouter de la musique grâce au dispositif pour écouteurs et aux boutons fixés sur l'accoudoir droit de chaque fauteuil.

L'organisation de l'espace et de la morphologie de ces trains configure un espace "ouvert», "public", mais en même temps formel et «individuel» étant donné la possibilité d'isolement (physique et acoustique), étant donné aussi que chaque place est dotée de plusieurs objets personnels (tablette, écouteurs, corbeille, etc.) qui transforment ce lieu en espace topique où l'on pourra vaquer à des occupations 
utiles ou plaisantes sans avoir l'impression de délaisser ses activités personnelles.

\section{INTERSUBJECTIVITÉS PRÉCONFECTIONNÉES}

Globalement, la comparaison des différents modèles de trains a permis d'élaborer quelques considérations à partir de la disposition des objets dans l'organisation interne de l'espace, un espace qui apparait comme une forme sémiotique produisant des discontinuités porteuses de signifiés. Nous avons identifié les configurations proxémiques les plus fréquentes qu'impliquent différentes manières possibles d'occuper l'espace et, par suite, d'encadrer l'interaction réciproque entre les passagers. Nous arrivons à une classification montrant comment différents types d'organisation spatiale déterminent autant de potentialités spécifiques d'interaction avec les objets et avec les autres passagers: par exemple, l'opposition entre espace "ouvert» (wagon) et espace "fermé» (compartiment) se transforme généralement, sur le plan du contenu, dans l'opposition espace «individuel» versus espace "collectif». Deux cas sont cependant différents: le train IR et l'ETR 450. Dans l'IR, l'espace peut être considéré comme "semi-ouvert" à cause de la disposition générale des fauteuils en microgroupes qui, en produisant une relation «sociale» et sociable entre passagers, se traduit en une dimension «semi-publique-collective». En ce qui concerne l'ETR 450, bien qu'il soit composé de wagons à rangées et non pas de compartiments (c'est donc un espace "ouvert»), il est - à cause de la disposition des objets - un espace «semi-fermé» mais «individuel» et, plus précisément sur le plan du contenu, «semi-privé-individuel». Il faut spécifier toutefois que l'espace que nous avons considéré comme individuel, collectif, privé, public, etc., n'est pas donné mais construit et perçu comme tel.

Comme on peut le remarquer, la seule opposition générale entre espace «ouvert» et espace «fermé» n'est pas suffisante pour rendre compte des configurations proxémiques possibles. Pour élaborer un meilleur système de classification, il est nécessaire en effet de tenir compte des différentes caractéristiques qui deviennent pertinentes en fonction du type de train. Entre autres, la morphologie des fauteuils (matériaux, formes, couleurs, tissus), la disposition des fauteuils, les tablettes (personnelles, que l'on peut extraire, ou centrées entre deux fauteuils), les accoudoirs (communs, rigides, larges ou étroits, etc.), les options présentes ou absentes à chaque place (cendrier, filodiffusione et prise pour les écouteurs, tablette, etc.). Dans le cas du train $I R$, en effet, l'espace «ouvert» est vécu comme «social» car les fauteuils créent des microgroupes et tous les éléments qui entourent et caractérisent l'espace individuel (tablettes personnelles, etc.) sont absents. L'ETR 450 est un train dépouillé, rationnel, avec un espace englobant; il ne stimule pas l'interaction entre les passagers et produit une certaine tension pour arriver rapidement au lieu prévu. Au contraire, les objets personnels dans les autres trains caractérisés par un espace "ouvert» privilégient chez les passagers une dimension émotive de «fermeture» et d'«individualité». Tous ces objets, ainsi que leurs qualités matérielles et leur disposition relationnelle, modalisent donc les passagers, sur le plan factitif, en fonction de leur voyage en train, en privilégiant la possibilité d'interaction sociale ou, au contraire, en favorisant l'isolement individuel. À partir de l'analyse de chaque train en tant que tel, il est en effet possible de faire émerger le parcours modal du passager qui, en fonction des objets qui l'entourent et de leur organisation, peut éprouver le désir d'interagir avec les autres (pouvoir-faire, vouloirfaire) ou bien se contenter des objets qui ont la capacité de l'occuper (tablette, écouteurs) en se sentant incapable de forcer les barrières dans l'espace qui, d'une certaine façon, l'isolent des autres (ne-paspouvoir-faire, ne-pas-devoir-faire) ${ }^{15}$. Dans chacun des cas analysés, les simples objets et l'ensemble des différents objets organisés deviennent des dispositifs modaux dissuasifs, persuasifs ou permissifs 16 .

Nous pouvons synthétiser graphiquement les problèmes abordés en classant chaque élément par opposition aux autres: 


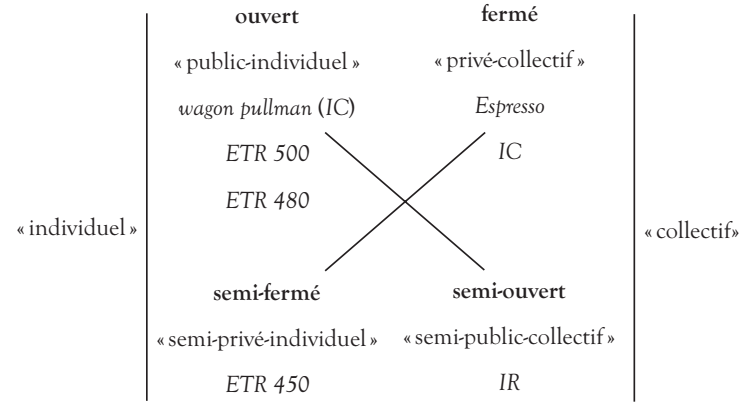

Il est clair qu'il ne s'agit pas d'un carré sémiotique dans lequel les contradictoires seraient "non-fermé» versus "non-ouvert», mais d'une opposition graduelle entre les deux types d'espaces «ouvert» et «fermé» et autant d'oppositions graduelles sur le plan du contenu. Les oppositions sur le plan du contenu s'articulent autour d'un espace perçu comme «individuel» dans la deixis positive, et "collectif» dans la deixis négative, mais avec certaines spécificités liées à l'effet de sens de chacun des quatre espaces identifiés dans les analyses: public-individuel; privé-collectif; semi-privé-individuel; semi-public-collectif ${ }^{17}$.

Traduisons maintenant les modalités de comportement prévues par les définitions suivantes:

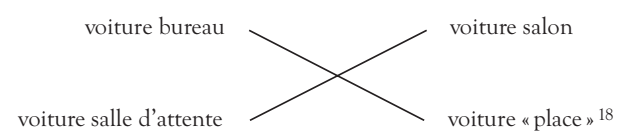

On peut conclure que l'ensemble des objets et leur disposition dans l'espace contribue à créer un milieu chargé d'effets de sens qui le rendent plus adapté à certaines activités qu'à d'autres (relaxation, lecture, travail, conversation, contacts sociaux, attente, etc.) grâce à une valeur modale qui, à chaque fois, le transforme en un espace caractérisé seulement eu égard aux autres espaces identifiables. Tout ceci, inévitablement, prescrit des modèles interactionnels qui, selon les cas, favorisent ou découragent le contact entre les passagers en entraînant des pratiques d'inclusion ou d'exclusion justement à partir du sens produit par la disposition et par les caractéristiques des nombreux objets. Objets qui, pris séparément, perdent une grande partie des potentialités sémiosiques qu'ils acquièrent, au contraire, de par leur cohabitation.

\section{N O TES}

1. Pour la définition de la modalité factitive, voir A. J. Greimas, Du sens

II, Paris, Seuil, 1983. Cf. aussi A.J. Greimas et J. Courtés, Sémiotique.

Dictionnaire raisonné de la théorie du langage, Paris, Hachette, 1979.

2. Sur ces objets, cf. B. Latour, Petites Leçons de sociologie des sciences, Paris, La Découverte, 1993.

3. Ici et par la suite, pour les définitions de marqueurs frontières et marqueurs centraux, voir E. Goffman, Relations in Public, New York, Basic Books, 1971.

4. Des exemples significatifs se trouvent chez J.-M. Floch, «Sémiotique et design. La scénographie du pouvoir dans le mobilier de haute direction ", Protée, vol.21, no 1, 1993 (analyse de la morphologie des bureaux des dirigeants) et chez B. Latour, op.cit., 1993 (analyse du "groom", du dos artificiel, des clés d'hôtels, etc.).

5. À ce propos, Barthes parle de parataxe (cf. L'Aventure sémiologique, Paris, Seuil, 1985).

6. Pour la définition d'espace topique et d'espace hétérotopique, cf. A.J. Greimas et J. Courtés, op. cit., 1979.

7. Simmel déjà («Die Großstädte und das Geistesleben », 1903, dans Brücke und Tür, Stuttgart, K.F. Koehler Verlag, 1957) avait indiqué comme «non-lieux» les espaces sociaux qui se transforment en se modelant sur le caractère «artificiel " et «intellectualisé » des métropoles. Sans citer Simmel, M. Augé définit comme «non-lieux» tous les espaces anonymes (routes, aéroports, centres commerciaux, transports, etc.) fréquentés quotidiennement par des "passagers » ou des «clients" qui, d'une certaine façon, s'y trouvent libérés de leur identité et des comportements habituels (M. Augé, Non-lieux, Paris, Seuil, 1992).

8. En nous inspirant d'U. Eco (Lector in fabula, Milan, Bompiani, 1979 et Sei passeggiate nei boschi narrativi, Milan, Bompiani, 1994), nous définissons l'Utilisateur Modèle comme une stratégie énonciative: c'est l'utilisateur-type que l'objet non seulement prévoit comme collaborateur mais qu'il cherche aussi à créer. Au contraire, l'Utilisateur Empirique est celui qui utilise effectivement l'objet mais peut aussi le faire avec des buts qui, apparemment ou non, n'ont aucun rapport avec l'objet même (utiliser un siège comme petite table, une chaussure comme marteau, etc.). Il existe évidemment des cas où utilisateur empirique et utilisateur modèle coïncident.

9. À ce propos, cf. A.J. Greimas, « Pour une sémiotique topologique», Sémiotique et sciences sociales, Paris, Seuil, 1976.

10. Cf. E.T. Hall, The Silent Language, New York, Doubleday, 1959 et The Hidden Dimension, New York, Doubleday, 1966.

11. Pour une description des modalités interactives des passagers du métro, cf. M. Augé, Un ethnologue dans le métro, Paris, Hachette, 1986. 12. Hall définit les proxèmes comme des configurations proxémiques répétées (E.T. Hall, «Sistema per la notazione del comportamento prossemico", dans Versus, Quaderni di studi semiotici, 2, 1972).

13. Cf. J. Ferreira, Métro. Le Combat pour l'espace. L'influence de

l'aménagement spatial sur les relations entre les gens, Paris, L'Harmattan, 1996. 14. Dispositif qui permet l'écoute par casques individuels de quelques canaux diffusant de la musique.

15. À ce propos, M. Hammad soutient que «l'espace vide est porteur de la modalité du pouvoir-faire. La matière solide n'est perçue, en première approche, qu'au titre de l'espace du ne-pas-pouvoir-faire " (dans "La privatisation de l'espace», Nouveaux Actes Sémiotiques, 4, 1989).

16. Cf. J. Fontanille, «Introduction», dans M. Hammad, op. cit., 1989. 17. Pour l'explication des dénominations qualitatives de public/privé associées aux dénominations quantitatives de collectif/individuel, voir A. Zinna, «Dinamiche di gruppo», dans Alfabeta, 1982.

18. Dans le sens italien de piazza. 